\title{
II. Ueber die chemische Zusammensetzung des Parisits und über ein neues Vorkommen desselben im Ravalli County, Montana.
}

\author{
Von \\ S. L. Penfield und C. H. Warren in New Haven, Conn. \\ (Hierzu 1 Textfigur.)
}

Vor Kurzem sandte IIcrr I. Cahn in New York an das mineralogische Laboratorium der Sheffield Scientific School zur Bestimmung einige Fxemplarc aus Montana, welche Krystalle von ungewöhnlichem Ansehen zeigten. Dieselben ergaben sich als dem seltenen Mineral Parisit angehörig, welches bisher nur in klcinen Mengen beobachtet worden war, und zwar am ursprünglichen Fundorte, den Smaragdgruloen des Musothales in Columbien, ferner sehr sparsam auf Ober-Arö im Langesundfjord, Norwegen (Brögger, dicse Zeitschr. 1890, 16, 6ə0), und ganz neuerdings bei Narsasuk in SüdGrünland, über welches letztere Vorkommen eine kurze Angabe von G. Flink in dem Berichte über seine im Sommer 1897 ausgeführte Reise mitgetheilt worden ist.

Da die chemische Zusammensetzung des Parisits noch nicht mit Sicherheit festgestcllt ist, so schien es nothwendig, nicht nur das neue Vorkommen mit dem eigenthümlichen Habitus seiner Krystalle zu beschreiben, sondern auch durch neue Analysen dic Frage nach der Formel dieses Minerals $\mathrm{zu}$ entschciden.

Die Parisitkrystalle von Montana sollen von »Pyrites \& im Ravalli County stammen und finden sich in einem feinen, kürnigen, lockeren, weissen Material, welches mit dem Fingernagel leicht zu l'ulver zerrieben werden kann. Diesc Matrix bestcht wesentlich aus Kieselsäure, Thonerde, Kalk und ctwas $\Lambda$ lkali und hat das $\Lambda$ nschen eines zersetzten Ryoliths oder Trachyts; seine eigentliche Natur wurde indess nicht näher untersucht. In 
dieser weissen Substanz sind Krystalle, von l'yrit und Parisit, meist cinzeln, zerstreul; manchmal ist ajoer der Parisit auf Pyrilkrystallen aufgewachsen und umhüllt sie thoilweise oder ganz. 1)er Pyrit krystallisirt in Pentagondodekï̈denn nil schmalen $\Lambda$ Jstumplungen du'ch lIexä̈der und Oktaëder, und die Grösse der Krystalle schwankt von mikroskopischer bis zu $3 \mathrm{~mm}$ Durelumesser. Jie millleren Dimensionen der l'arisilkrystalle betragen $1 \mathrm{~mm}$ Jurchm. und $10 \mathrm{~mm}$ Iäinge. Ihre Ilïuligkeil ist so gross, dass man cin bis zehn derselben auf zehn Quadralcentimeler Oberflïche des Multergesteins beobathlet. Im Allgemeinen zeigen sie den Jabilus eines lotizontal gestreiften lıcxagonalen Stengels, aus steilen l'yramidentlïehen in oscillatorischer Combination zusimmmengesetzt und am Ende durch deulliche, manchmal recht grosse Pyramiden begrenzl. Beistehende ligur, welche einen Theil eines derarligen Stengels mit einer Endigung durstellt, giclst eine deulliche Vorstellung von der Fintwickelung der Krystalle.

bis Flaichen, welche in oscillatorischer Combination vorherrschend die Slengel bilden, sind die der Pyramide $o\{20 \overline{2} 1\}$. Es konnten Messungen der ein- und ausspringenden Winkel mit dem Reflexionsgoniomeler ange-

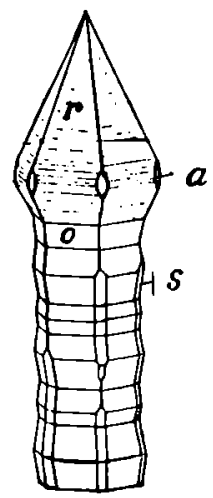
stellt werden, und diese sind in der weilerhin folgenden Tabelle angegeben. Einige der Kanten der Pyramide o sind durch eine Pyramide 2. Art $s\{11 \overline{2} 1\}$, ebenfalls in oscillalorischer Combination, abgestumpft; aber diese Form ist, wie aus der Figur ersichllich, im AlIgemeinen nicht zusammenhängend durch die ganze liïnge der Stengel, noch an allen Kanten ausgeljildet. Dic Pyramide, welche dic äusserste Endigung der Krystalle bildet, ist $r\{20 \overline{2} 3\}$, und dicse ist im Allgemeinen deutlich entwickelt, obgleich ihre Nittelkanten durch ein System feiner horizontaler Streifen etwas gerundet sind. Das in der Figur dargestellte Prisma 2. $\Lambda$ rt ist stets untergeordnct und fehll häufig ganz. Das Prisma 1. Ordnung $m\{10 \overline{1} 0\}$ wurde in deutlichen Fliichen nicht beol)achtet, aber die oscillatorische Combination der oljeren und unteren Pyramidenflächen bewirkt oft das Muftreten gestreifter und gerundeler Flïchen, welche nahezu dic Orientirung der Flächen jenes Prismas besitzen. Gelegentlich lanfen die Stengel auch am Ende spitz zu, ohne dass dasclbst cine dickere lyramide aufgesetzt crscheint. Von den sonach J)oluachteten Formen $a\{11 \overline{2} 0\}, r\{20 \overline{2} 3\}, o\{20 \overline{2} 1\}, s\{11 \overline{2} 1\}$ und $m$ ? $\{10 \bar{T} 0\}$ ist das Prisma $a$ neu. Jie gemessenen Winkel sind im Folgenden zusammengestell mit den aus Des Cloizcaux's ${ }^{1}$ ) Axenverhältniss $a: c=$ 1 : 3,289.1 berechneten Werthen:

1) Man. d. Minéral. 1871, 2, 162. 


\begin{tabular}{lrrr} 
& Bcobachtet: & \multicolumn{2}{c}{ Bcrechnel: } \\
$a: a=(11 \overline{2} 0):(\overline{1} 2 \overline{1} 0)=59^{0} 5 h^{\prime}$ & $60^{0} 0^{\prime}$ \\
$r: r=(20 \overline{2} 3):(02 \overline{2} 3)$ & 5325 & 55251 \\
$r: r=(20 \overline{2} 3):(\overline{2} 023)$ & 13722 & 136 & 54 \\
$s: s=(11 \overline{2} 1):(11 \overline{2} T)$ & 1723 & 1718 \\
$o: o=(20 \overline{2} 1):(20 \overline{2} \bar{T})$ & 1310 & 14 & 0
\end{tabular}

l'ür die chemische Analyse wurde das bestmögliche Material erhalten durch Auslesen der Krystalle aus der Matrix und Reiben derselben zwischen den Fingern zur Beseitigung des lose anhängenden Materials. Die Krystalle urnschliessen, wic erwähnt, ein wenig Pyrit und sind nicht frei von Sprüngen, auf denen etwas kieseliges Material aljgesetzt erscheint. Diese Verunreinigungen betragen etwa $6 \%$. Das Material war von fast gleichförmiger, gelbbrauner Farbe.

Dic Methode der Analyse war die folgende: Das Kohlendioxyd wurde durch Lösen des Minerals in Salzsïure und Aufsaugen des entwickelten Gases in gewogenem Kaliapparate bestimmt. Zur Fluorbestimmung wurde cine andere Portion des Materials mit Natriumcarbonat und ciner gewogenen Menge Kieselsüure geschmolzen und das Fluor getrennt und bestimmt nach der von Penfield und Minor modificirten Berzelius'schen Methode ${ }^{1}$ ). Die Erden wurden vom Calcium durch wiederholte Fällungen mit $\Lambda \mathrm{m}$ moniak getrennt, dann in Oxalute umgewandelt, geglüht und als Oxyde gewogen. Die Menge des Ceriumoxyds $\mathrm{Ce}_{2} \mathrm{O}_{3}$ ergab sich durch Zersetzung der geglühten Oxyde $\left(\mathrm{CeO}_{2}+\mathrm{La}_{2} \mathrm{O}_{\mathrm{s}}+\mathrm{Di}_{2} \mathrm{O}_{\mathrm{i}}\right)$ mit Schwefelsäure, der etwas Oxalsäure zugesetzt war, und Wägung des Kohlendioxyds, welches durch das hühere Oxyd des Ceriums, entsprechend der Gleichung $2 \mathrm{CeO}_{2}+\mathrm{H}_{2} \mathrm{C}_{2} \mathrm{O}_{4}$ $+\mathrm{Ce}_{2} \mathrm{O}_{3}+2 \mathrm{CO}_{2}+\mathrm{H}_{2} \mathrm{O}$, frei gemacht wird. Das Molekulargewicht der gemischten Oxyde $(\mathrm{Ce}, \mathrm{La}, \mathrm{Di})_{2} \mathrm{O}_{3}$ wurde durch Umwandlung ciner gewogenen Menge derselben in Sulfat bestimmt und zu 328,2 gefunden. Die Resultate der Analyse sind auf S. 7 unter I angegeben.

Da der Betrag der Verunreinigungen im Parisit von Montana die Berechnung der Formel etwas unsicher erscheinen liess, so wurde auch eine Analyse des Minerals vom Musothal angestellt. Dazu diente ein ungewöhnlich reines Material, das Fragment eines Krystalls aus der Brush'schen Sammlung. Es ergab sich bei dieser $\Lambda$ nalyse, dass nicht der ganze Gehalt an Fluor durch einmaliges Schmelzen mit Natriumcarbonat und Kieselsäure und Aussüssen mit Wasser zu erhalten war; der Rückstand, cin zweites Mal mit Natriumcarbonat und cin wenig Kieselsüure geschmolzen, lieferte noch ungefihr $0,5 \%$ Fluor. Da diese Vorsicht bei der Analyse des Minerals von Montana nicht beobachtet worden war, so ist der Fluorgehalt

1) S. diese Zeilschr. 1894, 23, 322. 
dieser Analyse etwas zu niedrig ausgefallen. Das Molekulargewicht der gemischlen Oxyde von Cer, Lanthan und Didym ergab sich zu 328,4.

Die Resultate der von Warren ausgeführten Analysen sind im folgenden mit denjenigen der Analyse des l'arisits von Muso von Damour und Deville (Compt. rend. 1864, 59, 270) zusammengestellt.

I. II. III.

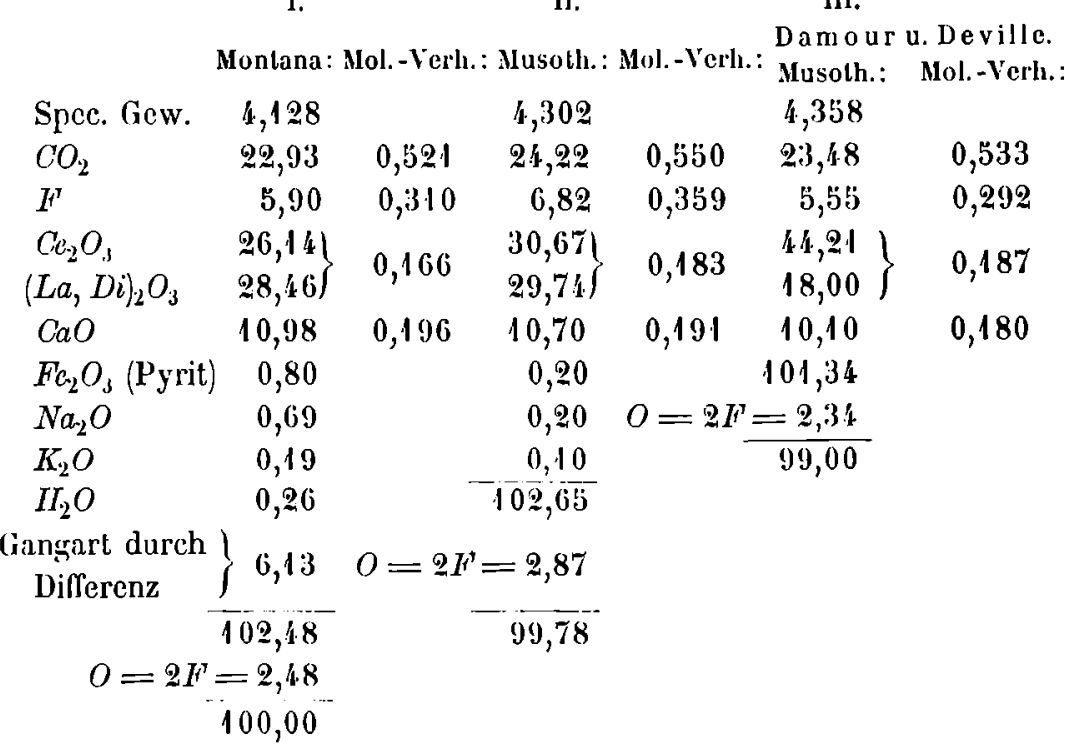

Aus vorstehenden $\Lambda$ nalysen ergeben sich die folgenden Verhältnisse:

$$
\mathrm{CO}_{2}: \mathrm{F}^{\prime}:(\mathrm{Ce}, \mathrm{La}, \mathrm{Di})_{2} \mathrm{O}_{\mathbf{3}}: \mathrm{CaO}
$$

I. $0,521: 0,310: 0,166: 0,196=3,00: 1,79: 0,96: 1,13$

II. $0,550: 0,359: 0,183: 0,191=3,00: 1,96: 1,01: 1,04$

III. $0,533: 0,292: 0,187: 0,180=3,00: 1,63: 1,05: 1,01$

J)ic neue Analyse des ungewöhnlich reinen Materials vom Musothal führt auf cin Verhältniss, welches sehr nahe $=3: 2: 1: 1$. Das Material von Montana war nicht so rein, daher das Verhältniss weniger befriedigend ist. ber Fluorgehalt des Minerals verursacht zweifellos, dass etwas Gangmaterial in Lösung geht und so das Calcium ein wenig zu hoch ausfällt, während der Fluorgehalt offenbar zu nicdrig bestimmt wurde, weil, wic oben angegeben, bei der Bestimmung dieses Bestandtheiles die Vorsicht, die Schmelzung mit Natriumcarbonat und Kiesclsïure zweimal vorzunehmen, unterlassen worden war. Jas von Damour und Deville erhaltene Verhültniss stimmt mit dem aus den neuen Analysen folgenden übercin mit $\Lambda$ usnahme desjenigen für das Fluor. Indessen muss angenommen werden, dass ihre Methode der Fluorbestimmung nicht einwurfsfrei ist, da sie keine directe Bestimmung dieses Elementes vorgenommen haben, sondern annahmen, dass beim Lösen des 
Mincrals in sehr verdünnter Chlorwasserstoffsüure das Fluor vollständig in Form der Fluoride von Calcium und Cerium getrennt werde und man es daher durch dic Differenz des Gewichtes dieser Fluoride und des Gewichtes von $C a$ und $C e$, welches sich aus der Analyse des unlüslichen Rückstandes ergiebt, finden könne.

Wenn man von den Müngeln der Analysen absieht, welche offenbar durch Ungenauigkeit der Fluorbestimmung verursacht sind, so weisen die aus den drei Analysen sich ergebenden Molckularverhältnisse somit darauf hin, dass $\mathrm{CO}_{2}, \mathrm{~F},(\mathrm{Ce}, \mathrm{La}, \mathrm{Di})_{2} \mathrm{O}_{3}$ und $\mathrm{CaO}$ in dem Verhältnisse 3:2:1:1 stehen. Bezcichnet man die dreiwerthigen Metalle der Ceriumgruppe mit $R$, so ist es wahrscheinlich, dass das Fluor mit diesen direct zu einem zweiwerthigen Radicale $R F$ verbunden und dass der Parisit ein Doppelcarbonat von der folgenden Constitution ist:

$$
2(R F) \mathrm{CO}_{3}+\mathrm{CaCO}_{3} \text { oder: }(\mathrm{RF})_{2} \mathrm{Ca}\left(\mathrm{CO}_{\mathrm{J}}\right)_{3} \text {. }
$$

Vorstchende Formeln sind, was das Verhältniss der einzelnen Bestandtheile betrifft, in Uebercinstimmung mit der von Groth (Tab. Uebers. d. Min. 1898, S. 61) vorgeschlagenen Formel

$$
(\mathrm{CaF})^{\prime}(\mathrm{CeF})^{\prime \prime} \mathrm{Ce}\left(\mathrm{CO}_{3}\right)_{3} \text {. }
$$

Groth gründete seine Formel auf die einzige, oben angeführte $\Lambda$ nalyse von Damour und Deville, indem er die richtige Annahme machte, dass der Verlust an Fluor durch den Mangel einer exacten Bestimmung dieses Bestandtheils verursacht sci. Gegenüber der doppelten Rolle, welche das Fluor nach der Auffassung des Genannten in der Verbindung spielt, nümlich als Bestandtheil des einwerthigen Radicals $(C a F)$ und des zweiwerthigen $(C e F)$, scheint es uns jedoch richtiger, nur eine $\Lambda$ rt der Verbindung dessclben, nämlich mit den Metallen $C e, L a, D i$ zu einem zweiwerthigen Radicalc $(R F)$, anzunchmen.

Min.-petr. Laboratorium der Shefficld Scientific School. 